\title{
Dissecting Patterns of Care in Patients With Variant Histology of Bladder Cancer and Lymph Node Invasion
}

\author{
Marco Bandini, ${ }^{1 *}$ Filippo Pederzoli, ${ }^{*}$ Andrea Necchi, ${ }^{2}$ Roger Li, ${ }^{3}$ Roberta Lucianò, ${ }^{4}$ Giuseppe Basile, ${ }^{\otimes} 1$ \\ Simone Scuderi, ${ }^{1}$ Riccardo Leni, ${ }^{1}$ Alberto Briganti, ${ }^{1}$ Andrea Salonia, ${ }^{1}$ Francesco Montorsi, \\ Andrea Gallina, ${ }^{1}$ Philippe E. Spiess ${ }^{3}$ \\ ${ }^{1}$ Unit of Urology, Urological Research Institute (URI), San Raffaele Hospital, Vita-Salute San Raffaele University, Milan, Italy ${ }^{2}$ Unit of Oncology, San Raffaele Hospital, \\ Vita-Salute San Raffaele University, Milan, Italy ${ }^{3}$ Moffitt Cancer Center and Research Institute, Tampa, United States ${ }^{4}$ Department of Pathology, IRCCS Ospedale \\ San Raffaele, Milan, Italy * Marco Bandini and Filippo Pederzoli equally contributed to the manuscript.
}

\section{Abstract}

Objectives Lymph node invasion (LNI) is related to long-term survival in patients with muscle-invasive bladder cancer. However, in the case of variant histology $(\mathrm{VH})$, data on pelvic lymph node dissection (PLND) and LNI are sparse. We described the pattern of care of PLND in patients with VHs of bladder cancer, exploring predictors of LNI.

Methods Using the 2001-2016 SEER registry, 20767 bladder cancer patients who underwent PLND were identified. Included histological variants were pure urothelial carcinoma (UC), micropapillary UC, sarcomatoid UC, lymphoepithelioma-like UC, adenocarcinoma, sarcoma, giant and spindle cell carcinoma, squamous cell carcinoma (SCC), and neuroendocrine tumor. Uni- and multivariable logistic regression analyses tested for LNI predictors. Cox regression was used to test for predictors of overall mortality $(\mathrm{OM})$ among both LNI positive and LNI negative patients.

Results Overall, 2464 (11.9\%) harbored a VH. On multivariate analysis, only micropapillary UC was associated with higher risk $(\mathrm{OR}=3.39)$ of LNI. This association was maintained when only the subset of patients treated without perioperative chemotherapy were analyzed $(\mathrm{OR}=3.30)$. Similarly, higher $\mathrm{T}$ stage $(\mathrm{T} 2$ stage $\mathrm{OR}=2.24$; T3-4 stage $\mathrm{OR}=9.44)$ and the use of chemotherapy $(\mathrm{OR}=2.29)$ were associated with a higher risk of LNI. Among patients with LNI $(5299,25.5 \%)$, SCC $(\mathrm{HR}=1.87)$, T3-4 stage $(\mathrm{HR}=1.94)$, age at diagnosis $(\mathrm{HR}=1.01)$ and geographic region (south) $(\mathrm{HR}=1.22)$ were predictors of higher risk of OM. Conversely, chemotherapy $(\mathrm{HR}=0.69)$ and number of removed LN $(\mathrm{HR}=-0.99)$ were associated with lower risk of OM. Finally, in a subgroup of patients without LNI, sarcomatoid UC $(\mathrm{HR}=1.58)$ and giant and spindle cell carcinoma $(\mathrm{HR}=1.83)$ were the only $\mathrm{VH}$ predictors of OM.

Conclusions We described different patterns of care in patients with VHs of bladder cancer. Micropapillary UC was an independent risk factor for LNI. Among patients harboring LNI, those with SCC VH had higher OM compared to pure UC. Conversely, sarcomatoid UC and giant and spindle cell carcinoma were predictors of OM in patients without nodal involvement.

\section{Key Words}

Bladder cancer, histological variants, lymph node dissection, lymph node metastasis

\section{Competing Interests}

None declared.

\section{Article Information}

Received on April 18, 2021

Accepted on July 4, 2021

Soc Int Urol J.2021;2(5):282-298

DOI: 10.48083/DHHV3158 


\section{Abbreviations}

LNI lymph node invasion

MIBC muscle-invasive urothelial cancer

OM overall mortality

PLND pelvic lymph node dissection

SCC squamous cell carcinoma

SEER Surveillance, Epidemiology, and End Results

TURB transurethral resection of the bladder

UC urothelial carcinoma

\section{Introduction}

Lymph node metastases may occur in up to one fourth of patients with muscle-invasive urothelial cancer of the bladder (MIBC)[1], and their presence is an important indicator of long-term survival and cancer recurrence[2-4]. Therefore, radical cystectomy with pelvic lymph node dissection (PLND) is the standard treatment for MIBC $[5,6]$.

While general agreement exists with respect to pure urothelial carcinoma, fragmentary evidence suggests caution in generalizing these findings to all the other histological variants of urothelial carcinoma and nonurothelial histology[7-11]. Histological variants are present in up to $25 \%$ of cystectomy specimens, and they have been traditionally associated with aggressive behavior[12-14]. Here, paucity of data about the use and the role of the PLND and the prevalence of lymph node metastases in these histological subtypes makes it difficult to determine a standard of care.

Given the lack of consensus, the European Association of Urology (EAU) partnered with the European Society of Medical Oncology (ESMO) and provided recommendations to guide specialists in this setting[7]. However, novel studies must address several gaps in the management of variant histology bladder cancer, including the different patterns of lymph node involvement according to the specific histology.

Using a population-based registry, we provided a description of the PLND patterns and the prevalence of lymph node invasion (LNI) in bladder cancer of urothelial and variant histology. Additionally, we explored potential predictors of lymph node metastasis.

\section{Materials and Methods}

\section{Study Population}

Within the Surveillance, Epidemiology, and End Results (SEER) database (2001-2016)[15], we selected patients aged 18 years or older with histologically confirmed bladder cancer (International Classification of Disease for Oncology [ICD-O] site codes C670-679), excluding patients with urachal tumors (ICD-O site code C677) (Supplementary Figure 1; available at siuj.org). For the purpose of our analysis, we focused on patients with bladder cancer and non-systemic metastasis (T0-4N0-3) who underwent any form of treatment to the primary tumor, as well as PLND, and who harbored the following primary bladder histological variants (Supplementary Table 1; available at siuj.org): urothelial carcinoma (UC), micropapillary UC, lymphoepithelioma-like UC, sarcomatoid UC, adenocarcinoma, squamous cell carcinoma (SCC), giant and spindle cell carcinoma, neuroendocrine tumor and sarcoma[16]. These selection criteria yielded an initial study population of 20767 patients.

\section{Variables Definition}

The variables considered included variant histology together with the presence of LNI. Study covariates included age at the time of bladder cancer diagnosis, sex, race (White, Black, Hispanic, Asian, and other), marital status (married, never married, previously married, unknown), geographical region (West, Midwest, North-East, South), socioeconomic status (high versus low), administration of perioperative chemotherapy (either neoadjuvant or adjuvant), local treatment modality (radical cystectomy [RC], partial cystectomy, transurethral resection of the bladder [TURB] only, radiotherapy, trimodal therapy [TMT], no local treatment, other/unknown), T stage (T0/x, T<2, T2, T3-4), tumor grade (G1-4, unknown), number of lymph nodes removed (NNR), number of positive lymph nodes (NPN), and lymph node density (LN density).

\section{Statistical Analysis}

Descriptive statistics includes frequencies and proportions for categorical variables. Means, medians, and ranges were reported for continuously coded variables. Chi-square was used to test the statistical significance in proportions' differences. The $t$ test and Kruskal-Wallis test examined the statistical significance of differences in means and medians[17]. Univariable and multivariable logistic regression analyses were used to test for predictors of LNI among patients who received PLND among several covariates. Sub-analyses focused on patients treated with RC. Univariable and multivariable Cox regression analyses were used to test for predictors of overall mortality (OM) among patients with LNI. Moreover, we performed sensitivity analyses focusing on patients who did not receive perioperative chemotherapy to mitigate the possible effect of either neoadjuvant or adjuvant chemotherapy treatments on both LNI and OM prediction. Finally, univariable and multivariable Cox regression analyses were also used to test for predictors of OM among patients without nodal disease (N0). $P$-values were adjusted using the conservative Bonferroni correction method, which 
multiplies the raw $P$-values by the number of tests. For all statistical analyses, $\mathrm{R}$ software environment (version 3.6.1) was used. All tests were 2-sided with a level of significance set at $P<0.05$.

\section{Results}

\section{Overall descriptive characteristics}

Overall, we identified 20767 patients diagnosed with bladder cancer between 2001 and 2016 who underwent PLND (Table 1). Among them, 18303 (88.1\%) patients had UC, 889 (4.3\%) SCC, 592 (2.9\%) adenocarcinoma, 382 (1.8\%) neuroendocrine tumor, 283 (1.4\%) sarcomatoid UC, 177 (0.9\%) micropapillary UC, 85 (0.4\%) giant and spindle cell carcinoma, $38(0.2 \%)$ lymphoepithelioma-like UC and $18(<0.1 \%)$ sarcoma. The vast majority of patients were diagnosed with $\mathrm{T} \geq$ 2 disease $(17843,85.9 \%)$, without major differences across the histologic subtypes. $\mathrm{RC}$ represented the main primary treatment in all groups. Among non-RC primary treatments, a high percentage $(132,22.3 \%)$ of partial cystectomies was performed in adenocarcinoma patients (Figure 1). Perioperative chemotherapy was evenly administered in the different histologic subtypes, with patients with lymphoepitheliomalike UC $(25,65.8 \%)$, neuroendocrine tumor (250, $65.4 \%)$ and micropapillary UC $(91,51.4 \%)$ being most frequently treated with chemotherapy. The median number of removed lymph nodes ranged from 11 to 19 in all the study groups (Figure 2): patients with lymphoepithelioma-like UC had the highest number of lymph nodes removed (median 19, interquartile range [IQR]: 7-26), while patients with giant and spindle cell carcinoma had the lowest (11, 5-19).

\section{LNII Cohort}

In the study population, LNI was reported in 5299 (25.5\%) patients (Supplementary Table 2; available at siuj.org), specifically 88 (49.7\%) micropapillary UC, 117 (30.6\%) neuroendocrine tumor, 175 (29.6\%) adenocarcinoma, 11 (28.9\%) lymphoepithelioma-like UC, 72 (25.4\%) sarcomatoid UC, 4596 (25.1\%) UC, 220 (24.7\%) SCC, 17 (20.0\%) giant and spindle cell carcinoma, 3 (16.7\%) sarcoma. Patients with LNI had a higher stage disease (T3-4 stage $77.0 \%$ versus $41.6 \% ; P<0.001$ ) and were more frequently treated with chemotherapy $(55.1 \%$ versus $32.9 \%$; $P<0.001)$ than patients without malignant nodal involvement (Table 2). Among different histological variants, UC (3.1\%) and micropapillary UC (3.4\%) presented the highest rates of LNI in non-muscleinvasive disease. Compared with UC (19.9\%), all the other histological variants had lower rates of LNI in T2 disease, with the exception of micropapillary UC, which presented higher (27.3\%) rates of LNI (Supplementary Table 2; available at siuj.org). At multivariate logistic regression analysis (Table 3), only micropapillary UC was associated with a higher risk $(\mathrm{OR}[95 \% \mathrm{CI}]=3.39$ [2.43-4.74]; $P<0.001$, Bonferroni adjusted $P<0.001$ ) of LNI among the histological variants. Similarly, higher $\mathrm{T}$ stage (T2 stage OR [95\%CI] $=2.24[1.88-2.69]$, $P<0.001$, Bonferroni adjusted $P<0.001$; T3-4 stage OR $[95 \% \mathrm{CI}]=9.44$ [7.98-11.2], $P<0.001$, Bonferroni adjusted $P<0.001$ ), and the use of chemotherapy (OR $[95 \% \mathrm{CI}]=2.29[2.13-2.46] ; P<0.001$, Bonferroni adjusted $P<0.001$ ) were associated with a higher risk of LNI. Analyses restricted to the population who received $\mathrm{RC}$ are shown in Supplementary Table 3 (available at siuj. org).

\section{Sensitivity analysis of LNI among patients without} perioperative chemotherapy treatment

Overall, 12761 (61.4\%) patients did not receive either neoadjuvant or adjuvant chemotherapy. Particularly, 11187 (87.7\%) patients had UC, 683 (5.4\%) SCC, 402 (3.2\%) adenocarcinoma, $132(1.0 \%)$ neuroendocrine tumor, 182 (1.4\%) sarcomatoid UC, 86 (0.7\%) micropapillary UC, $63(0.5 \%)$ giant and spindle cell carcinoma, $13(0.1 \%)$ lymphoepithelioma-like UC and 13 (0.1\%) sarcoma, as shown in Supplementary Table 4 (available at siuj.org). Among these, LNI was reported in 2380 (18.6\%) patients (Supplementary Table 5; available at siuj.org). At multivariate logistic regression analysis (Supplementary Table 6; available at siuj.org), both micropapillary UC $(\mathrm{OR}[95 \% \mathrm{CI}]=3.30$ [2.01-5.37]; $P<0.001$, Bonferroni adjusted $P<0.001$ ) and higher $\mathrm{T}$ stage (T2 stage OR $[95 \% \mathrm{CI}]=3.31[2.54-4.37] ; P<0.001$, Bonferroni adjusted $P<0.001$; T3-4 stage OR $[95 \% \mathrm{CI}]=15.39$ [12-20.1]; $P<0.001$, Bonferroni adjusted $P<0.001$ ) were confirmed to be associated with a higher risk of LNI.

\section{Overall Mortality Data}

When considering patients with LNI, median OM rate was 24 months (IQR 23-25) with a median follow-up of 22 months (IQR 9-42.7) regardless of histology variant. OM rates according to variant histology in patients with LNI is reported in Supplementary Table 7 (available at siuj.org). Among patients with LNI (Table 4), predictors of higher risk of OM were SCC (hazard ratio, HR [95\%CI] = 1.87 [1.56-2.24]; $P<0.001$, Bonferroni adjusted $P<0.001$ ), histology, T3-4 stage (HR [95\%CI] = 1.94 [1.52-2.47]; $P<0.001$, Bonferroni adjusted $P=0.03$ ), age at diagnosis $(\mathrm{HR}[95 \% \mathrm{CI}]=1.01[1.01-1.01] ; P<0.001$, Bonferroni adjusted $P<0.001)$, never married $(\mathrm{HR}[95 \% \mathrm{CI}]=1.24$ [1.11-1.39]; $P<0.001$, Bonferroni adjusted $P<0.001)$, and South Region (HR [95\%CI] $=1.22[1.10-1.35] ; P<0.001$, Bonferroni adjusted $P<0.001)$. Conversely, chemotherapy $(\mathrm{HR}[95 \% \mathrm{CI}]=0.69$ [0.64-0.74]; $P<0.001$, Bonferroni adjusted $P<0.001$ ) and number of removed LN (HR $[95 \% \mathrm{CI}]=0.99[0.99-0.99] ; P<0.001$, Bonferroni adjusted $P=0.03$ ) were associated with lower risk of OM. 
Analyses restricted to the population who received RC are shown in Supplementary Table 8 (available at siuj.org).

\section{Sensitivity analysis of overall mortality among patients without perioperative chemotherapy treatment}

Among patients with LNI and no perioperative chemotherapy treatment $(\mathrm{n}=2380$, Supplementary Table 9; available at siuj.org), SCC $(\mathrm{HR}[95 \% \mathrm{CI}]=2.06$ [1.64-2.59]; $P<0.001$, Bonferroni adjusted $P<0.001$ ) was confirmed to be the only histology variant predictor of OM. Moreover, number of removed LN (HR $[95 \% \mathrm{CI}]=0.98$ [0.98-0.99]; $P<0.001)$, Bonferroni adjusted $P<0.001$ ) was associated with lower risk of OM.

\section{Sensitivity analysis of overall mortality among patients without LNI}

When considering patients without lymph node invasion ( $\mathrm{n}=15$ 468, Supplementary Table 10; available at siuj.org), sarcomatoid UC (HR [95\%CI] = 1.58 [1.281.94]); $P<0.001$, Bonferroni adjusted $P=0.001$ ) and giant and spindle cell carcinoma $(\mathrm{HR}[95 \% \mathrm{CI}]=1.83$ [1.29-2.59]; $P<0.001$, Bonferroni adjusted $P=0.026$ ) were the only histology variants predictors of OM, as compared to the LNI cohort. Conversely, both chemotherapy $(\mathrm{HR}[95 \% \mathrm{CI}]=0.89[0.83-0.95] ; P<$ 0.001 , Bonferroni adjusted $P=0.015$ ) and number of removed LN (HR [95\%CI] $=0.99$ [0.99-0.99]; $P<0.001$, Bonferroni adjusted $P<0.001$ ) were confirmed to be associated with lower risk of OM.

\section{Discussion}

In this study, we explored the patterns of care associated with the diagnosis of a non-pure urothelial carcinoma of the bladder in the SEER database. We focused our attention on the LNI distribution according to the histological diagnosis in PLND patients, eventually exploring potential predictors of lymph node metastasis.

In general, histological variants are considered more aggressive than pure $\mathrm{UC}$, which requires an aggressive therapeutic management even in case of non-muscleinvasive disease. For instance, the micropapillary, plasmacytoid and sarcomatoid variants of urothelial carcinoma have a worse prognosis than comparable high-grade, pure UC[18-21]. Therefore, the European Association of Urology (EAU) guidelines have included them in the highest risk group of non-muscle-invasive bladder cancer, for which radical cystectomy +/neoadjuvant treatments should be considered[19]. Our study confirmed the aggressiveness of some variants in terms of overall mortality when considering patients with LNI, reporting a higher OM risk for SCC among all the VHs considered, regardless of perioperative chemotherapy treatment. Conversely, among patients without LNI, sarcomatoid UC and giant and spindle cell carcinoma were associated with higher OM risk. Moreover, the survival advantage for bladder cancer patients linked to lymphadenectomy at the time of radical surgery has been shown by several studies[22], including a registry-based analysis[23], and it is supported by the fact that up to $25 \%$ of patients with organ-confined muscle-invasive bladder cancer have metastatic nodal disease[24].

It is still debated whether the presence of histological variants affects the incidence of lymph node metastasis, potentially affecting progression and survival. Kim et al. analyzed the outcomes of a cohort of 132 patients with bladder cancer showing squamous differentiation, 41 with glandular differentiation, and 13 with both variants[25]. The presence of differentiations, compared with a control cohort of pure UC patients, was associated with a higher rate of extravesical disease (pT3-T4 stage $70 \%$ versus $38 \% ; P<0.001$ ) and lymph node involvement ( $20 \%$ versus $15 \% ; P=0.05$ ), but it did not affect 10 -year cancer-specific survival (52\% versus $51 \%$; $P=0.71$ ). Similarly, a long-term analysis of a cohort of 52 patients with nested variant reported a positive association between the nested variant and the presence of locally advanced disease and lymph node metastases[26]. Nevertheless, the presence of nested variant did not influence either 10-year recurrence-free survival $(80 \%$ versus $83 \% ; P=0.46)$ or cancer-specific survival ( $41 \%$ versus $46 \%$; $P=0.75$ ), when compared with pure UC patients. Another recent cohort study[27] of 525 bladder cancer patients treated with open radical cystectomy at a single tertiary center reported a histological variant in 131 patients $(25.0 \%)$. Their data suggested that the presence of a variant histology was associated with a more advanced and biologically aggressive malignancy, including a higher frequency of lymphovascular invasion and nodal disease at radical cystectomy. Conversely, other studies did not find an association between histological variants and LNI, as reported by Marks et al. in their cohort of 138 patients with nodal metastases [28]. A variant histology was present in 96 patients, and the presence of variant histology was not associated with either the presence of lymph node metastases or extranodal extension. In our analysis, we found that the presence micropapillary UC was an independent risk factor for LNI after multivariate correction, and also after accounting for the possible effect of perioperative chemotherapy on nodal disease status. Micropapillary morphology in solid malignancies is known to have a distinct propensity for lymphovascular invasion, which can lead to a higher rate of LNI compared with the non-micropapillary histological variants $[29,30]$. Therefore, these observations suggest the need for further studies involving an accurate analysis of the biological, pathological, and clinical factors of the urothelial and non-urothelial bladder cancer variants 


\section{TABLE 1.}

Descriptive characteristics of the overall population of study $(n=20767)$ according to the different histological subtypes
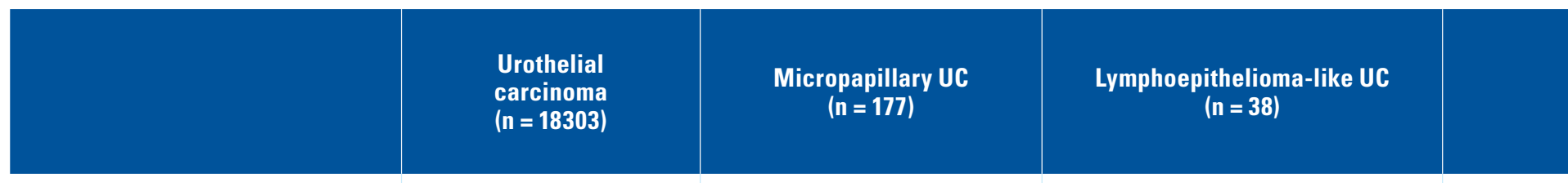

Age at diagnosis, median (IOR)

$68(61-76)$

$70(61-76)$

$68(62-75)$

Female sex, $n(\%)$

4163 (22.7)

31 (17.5)

9 (23.7)

\section{Race, n $1 \%$ \\ White \\ Black \\ Hispanic \\ Asiatic \\ Other}

$1055(5.8)$

$1158(6.3)$

$828(4.5)$

$97(0.5)$

$153(86.4)$
$5(2.8)$
$8(4.5)$
$10(5.6)$
$1(0.6)$

28 (73.7)

$4(10.5)$

2 (5.3)

$4(10.5)$

$0(0)$

\section{Socioeconomic status, n (\%)}

High

$9144(49.9)$

$18(47.4)$

Low

$9159(50.1)$

$91(51.4)$

20(52.6)

Region, n (\%)

West

Midwest

North-East

South

\section{Marital status, $\mathrm{n}(\%)$}

Married

Never married

Previously married

Unknown

Treatment, n (\%)

Radical cystectomy

Partial cystectomy

TURB only

Radiotherapy

TMT

No local treatment

Other/Unknown

\begin{tabular}{c|c}
$9927(54.2)$ & $84(47.5)$ \\
\hline $2104(11.5)$ & $30(16.9)$ \\
$3146(17.2)$ & $16(9)$ \\
\hline $3126(17.1)$ & $47(26.6)$
\end{tabular}

$117(66.1)$

$17(9.6)$

$36(20.3)$

7 (4)
28 (73.7)

$6(15.8)$

$4(10.5)$

$0(0)$
24 (63.2)

$3(7.9)$

$5(13.2)$

$6(15.8)$
33 (86.8)

2 (5.3)

2 (5.3)

$0(0)$

$0(0)$

$0(0)$

$1(2.6)$

CT: chemotherapy; IQR: interquartile range; LN: lymph node; LNI: lymph node invasion; NNR: number of lymph nodes removed; TMT: trimodal therapy; TURB: transurethral resection of the bladder; UC: urothelial carcinoma 

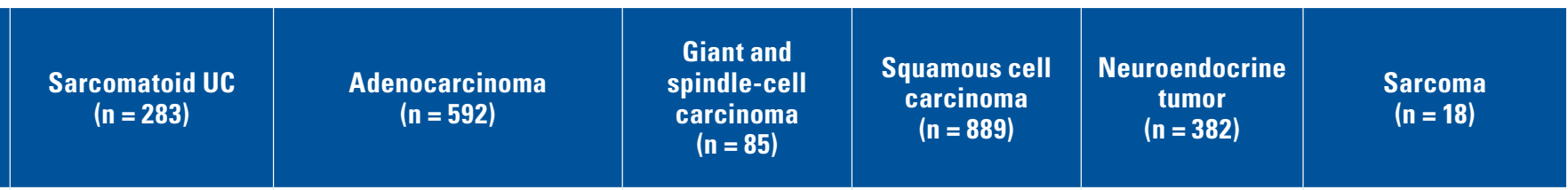

$69(61-76)$

$63(54-71)$

$71(59-77)$

$68(58-76)$

$68(61-75)$

75 (61-78)

91 (32.2)

226 (38.2)

27 (31.8)

439 (49.4)

70 (18.3)

6 (33.3)

\begin{tabular}{c|c}
\hline $225(79.5)$ & $410(69.3)$ \\
\hline $20(7.1)$ & $81(13.7)$ \\
\hline $19(6.7)$ & $59(10)$ \\
\hline $18(6.4)$ & $36(6.1)$ \\
\hline $1(0.4)$ & $6(1)$ \\
\hline
\end{tabular}

\begin{tabular}{c|c}
\hline $70(82.4)$ & $707(79.5)$ \\
$3(3.5)$ & $75(8.4)$ \\
\hline $8(9.4)$ & $74(8.3)$ \\
\hline $3(3.5)$ & $26(2.9)$ \\
$1(1.2)$ & $7(0.8)$
\end{tabular}

319 (83.5)

$15(3.9)$

27 (7.1)

$19(5)$

$2(0.5)$

14 (77.8)

1 (5.6)

3 (16.7)

$0(0)$

$0(0)$

\begin{tabular}{l|l}
\hline $131(46.3)$ & $314(53)$ \\
\hline $152(53.7)$ & $278(47)$
\end{tabular}

47 (55.3)

442 (49.7)

38 (44.7)

$447(50.3)$

192 (50.3)

190 (49.7)

12 (66.7)

6 (33.3)

\begin{tabular}{c|c}
\hline $129(45.6)$ & $327(55.2)$ \\
\hline $45(15.9)$ & $47(7.9)$ \\
\hline $48(17)$ & $112(18.9)$ \\
\hline $61(21.6)$ & $106(17.9)$
\end{tabular}

\begin{tabular}{c|c}
\hline $46(54.1)$ & $463(52.1)$ \\
\hline $6(7.1)$ & $103(11.6)$ \\
\hline $19(22.4)$ & $155(17.4)$ \\
\hline $14(16.5)$ & $168(18.9)$ \\
\hline
\end{tabular}

204 (53.4)

$38(9.9)$

13 (72.2)

74 (19.4)

$0(0)$

66 (17.3)

$3(16.7)$

2 (11.1)

175 (61.8)

27 (9.5)

73 (25.8)

8 (2.8)

\begin{tabular}{c|c}
\hline $254(89.8)$ & $389(65.7)$ \\
\hline $14(4.9)$ & $132(22.3)$ \\
\hline $3(1.1)$ & $13(2.2)$ \\
$0(0)$ & $0(0)$ \\
$0(0)$ & $8(1.4)$ \\
$0(0)$ & $3(0.5)$ \\
$12(4.2)$ & $47(7.9)$
\end{tabular}

345 (58.3)

102 (17.2)

118 (19.9)

27 (4.6)
45 (52.9)

12 (14.1)

24 (28.2)

4 (4.7)
493 (55.5)

124 (13.9)

242 (27.2)

30 (3.4)

\section{0 (68.1)}

46 (12)

63 (16.5)

13 (3.4)
12 (66.7)

2 (11.1)

4 (22.2)

$0(0)$ 


\section{TABLE 1.}

Descriptive characteristics of the overall population of study ( $n=20767$ ) according to the different histological subtypes, Cont'd
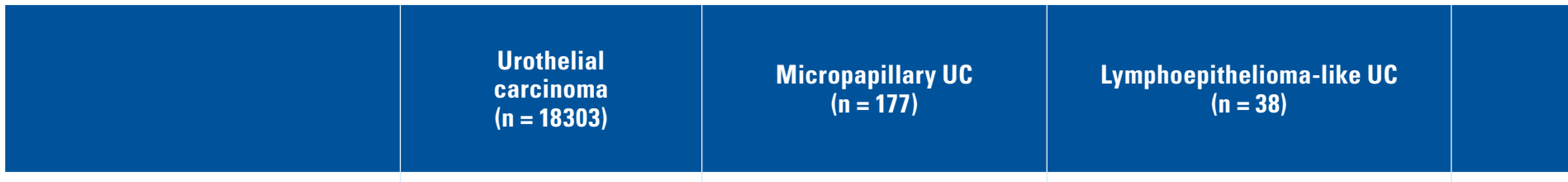

\section{Perioperative CT, n (\%) \\ Yes \\ No/Unknown}

$7116(38.9)$

$11187(61.1)$
$91(51.4)$
25 (65.8)

$13(34.2)$

\section{T stage, $n(\%)$}

\begin{tabular}{|c|c|c|c|}
\hline TO/x & $110(0.6)$ & $0(0)$ & $0(0)$ \\
\hline $\mathrm{T}<2$ & 2563 (14) & $23(13)$ & $4(10.5)$ \\
\hline T2 & 6694 (36.6) & 64 (36.2) & 12 (31.6) \\
\hline T3-4 & 8936 (48.8) & $90(50.8)$ & $22(57.9)$ \\
\hline
\end{tabular}

Grade, $\mathrm{n}(\%)$

\begin{tabular}{|c|c|c|c|}
\hline $\mathrm{G} 1$ & $114(0.6)$ & $0(0)$ & $0(0)$ \\
\hline $\mathrm{G} 2$ & $614(3.4)$ & $2(1.1)$ & $0(0)$ \\
\hline G3 & $5577(30.5)$ & $39(22)$ & $8(21.1)$ \\
\hline G4 & 11169 (61) & $127(71.8)$ & $26(68.4)$ \\
\hline $\mathrm{Gx}$ & $829(4.5)$ & $9(5.1)$ & $4(10.5)$ \\
\hline
\end{tabular}

LNI, $\mathrm{n}(\%)$

4596 (25.1)

88 (49.7)

$11(28.9)$

NNR, median (IQR)

$12(6-22)$

$15(9-26)$

$19(7-26)$

LN density, mean

7.8

21

8.9

CT: chemotherapy; IQR: interquartile range; LN: lymph node; LNI: lymph node invasion; NNR: number of lymph nodes removed; TMT: trimodal therapy; TURB: transurethral resection of the bladder; UC: urothelial carcinoma

to define the best multidisciplinary (surgery, radiation therapy, chemotherapy, and innovative treatment strategies) approach for each histological subtype, with a particular emphasis on immunotherapy and targeted therapies[31-34].

It is clear that the time has come to gain a clear, ordered and practice-changing knowledge about these rare entities. Single- and small multi-institutional studies are generally unable to collect enough cases belonging to the different subtypes, potentially resulting in underpowered findings. Although retrospective registry-based analyses have access to greater numbers, they are frequently incomplete and suffer from the lack of or underreporting of variables that may have had an impact on patient outcomes. With respect to bladder cancer variants, the lack of centralized pathological review of the cases, as well as the possible under recognition of rare histological entities outside highvolume centers, may hamper the generalization of the study conclusions. For all these reasons, to further advance the knowledge in the field of bladder cancer, centralized initiatives are needed to create prospective, centralized, multicenter bladder cancer registries, like the BRaVeRY (BladdeR Variants RegistrY) protocol sponsored by the EAU Research Foundation[35]. With 

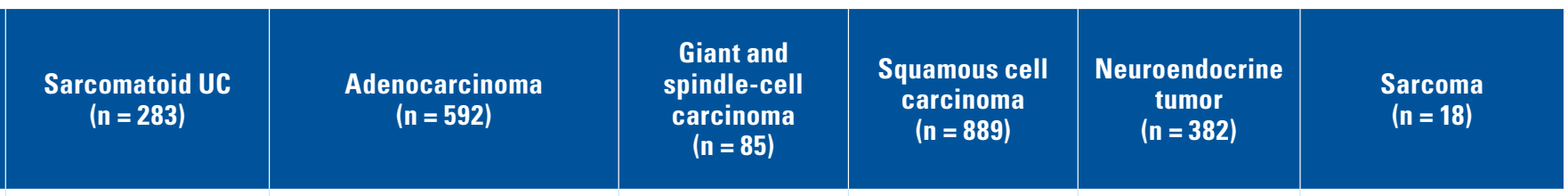

$101(35.7)$

$190(32.1)$

$22(25.9)$

$206(23.2)$

$250(65.4)$

5 (27.8)

182 (64.3)

402 (67.9)

63 (74.1)

683 (76.8)

132 (34.6)

13 (72.2)

\begin{tabular}{|c|c|}
\hline $2(0.7)$ & $6(1)$ \\
\hline $25(8.8)$ & $76(12.8)$ \\
\hline $81(28.6)$ & $137(23.1)$ \\
\hline $175(61.8)$ & $373(63)$
\end{tabular}

\begin{tabular}{|c|c|c|c|}
\hline $1(1.2)$ & $17(1.9)$ & $1(0.3)$ & $1(5.6)$ \\
\hline $4(4.7)$ & $52(5.8)$ & $36(9.4)$ & $3(16.7)$ \\
\hline $25(29.4)$ & $188(21.1)$ & $129(33.8)$ & $3(16.7)$ \\
\hline $55(64.7)$ & $632(71.1)$ & $216(56.5)$ & $11(61.1)$
\end{tabular}

\begin{tabular}{c|c}
$1(0.4)$ & $21(3.5)$ \\
\hline $3(1.1)$ & $149(25.2)$ \\
\hline $85(30)$ & $241(40.7)$ \\
$175(61.8)$ & $115(19.4)$ \\
$19(6.7)$ & $66(11.1)$ \\
\hline
\end{tabular}

\begin{tabular}{c|c|}
\hline $0(0)$ & $58(6.5)$ \\
\hline $1(1.2)$ & $344(38.7)$ \\
\hline $22(25.9)$ & $308(34.6)$ \\
\hline $48(56.5)$ & $136(15.3)$ \\
\hline $14(16.5)$ & $43(4.8)$ \\
\hline
\end{tabular}

\begin{tabular}{c|c}
$1(0.3)$ & $0(0)$ \\
$3(0.8)$ & $0(0)$ \\
\hline $101(26.4)$ & $4(22.2)$ \\
\hline $200(52.4)$ & $11(61.1)$ \\
\hline $77(20.2)$ & $3(16.7)$ \\
\hline
\end{tabular}

72 (25.4)

175 (29.6)

$17(20)$

220 (24.7)

117 (30.6)

3 (16.7)

13 (6-22)

$11(6-21)$

11 (5-19)

11 (6-20)

12 (7-23)

13 (6-23)

7.1

11.1

4.7

8.7

9.7

1.5

the creation of this multicenter registry, it will be possible to capture for the first time the therapeutic and surgical management of urothelial-histological variants and non-urothelial-histological variants in a real-life setting.

Despite its novelty, our study is not devoid of limitations. First, the presented retrospective SEER data[15] are influenced by inherent selection biases, as well as by the lack of variables that may have an impact on LNI, such as lymphovascular invasion, treatment choice, and patient eligibility for chemotherapy. One of the main limitations of our study is related to the impossibility of distinguishing between neoadjuvant and adjuvant chemotherapy treatment. Is well recognized, neoadjuvant chemotherapy could affect lymph node positivity rates, and patients who have neoadjuvant chemotherapy generally have more favorable oncological outcomes than patients who do not. As well, neoadjuvant treatments could be used differentially between histological variants. Nevertheless, micropapillary UC was confirmed to be the only histological variant predictor of LNI, even when patients who had not received any perioperative chemotherapy treatment were considered. Furthermore, SCC histology was found to be associated with a higher 


\section{FIGURE 1.}

Radar plot showing the main clinical and pathological characteristics in the study population according to histological variant

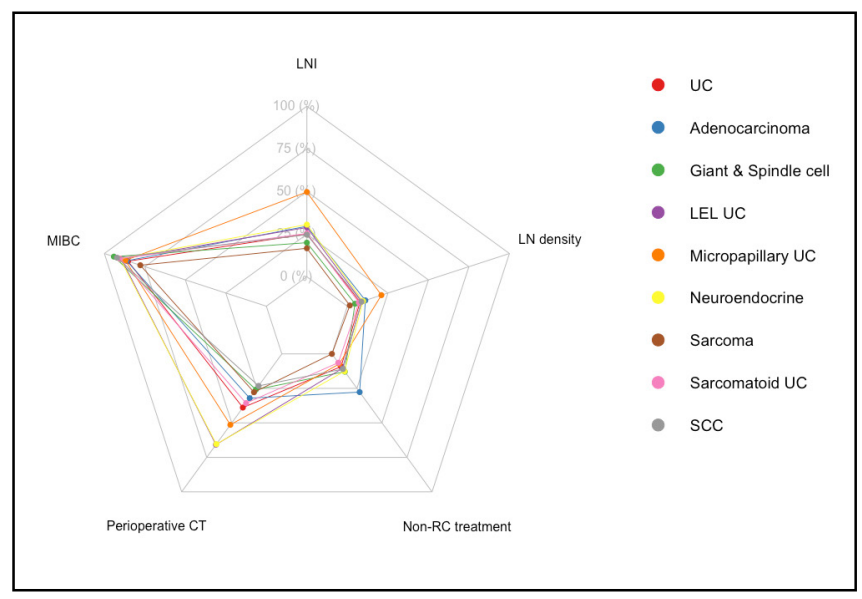

risk of OM than any of the other VHs considered, regardless of perioperative chemotherapy use, probably due to the inherent pathobiological behavior of this type of $\mathrm{VH}$. Moreover, the SEER database does not allow identification of the reason for PLND, and some patients may have received PLND for staging purposes or have had aborted procedures, which have also been reported for prostate cancer[36]. Further, the SEER database does not include all the histological variants of bladder cancer identified by the 2016 World Health Organization classification of tumors of the urinary tract[37]; our analysis is therefore limited to the coded entities. The SEER database does not provide a central pathological review, which may have an impact on the accuracy of the reported variants, as bladder cancer variants are commonly underrecognized outside tertiary, highvolume centers[38,39]. Additionally, the SEER database does not discriminate between the histologic diagnosis at transurethral resection of the bladder tumor and the histologic diagnosis at partial or radical cystectomy and does not provide a quantitative evaluation of the variant component compared with the total tumor volume. Finally, the high heterogeneity of local treatments considered may have affected the detection rate of variant histology included in this study.

\section{Conclusions}

We described different patterns of care according to the histological variants in bladder cancer using the SEER database. In particular, we analyzed the rate at which PLND was performed and the prevalence of LNI, as is

\section{FIGURE 2.}

Violin plot showing the number of removed lymph nodes (above) and wave plot (below) showing the lymph node density, according to histological variant

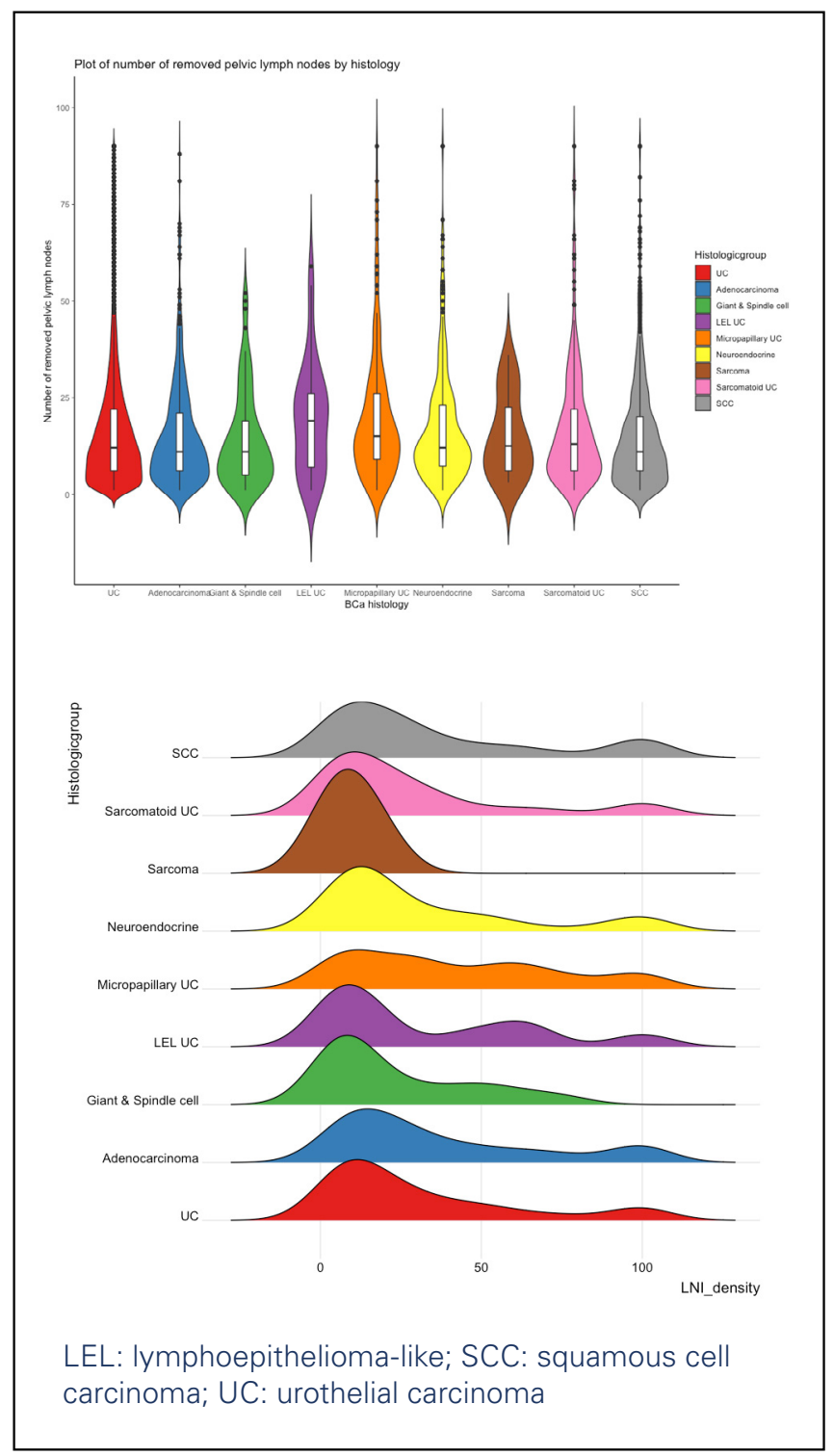

well as the potential predictors of each. We found that the presence of micropapillary UC was an independent risk factor for LNI after multivariate correction, regardless of perioperative chemotherapy treatment. Additionally, among patients with LNI, SCC histology was associated with higher OM compared with pure UC. Our analysis highlights the need for international collaborations to advance knowledge with respect to urothelial and non-urothelial bladder cancer variants. 
TABLE 2.

Descriptive characteristics according to the lymph node invasion status in the study population $(n=20767)$
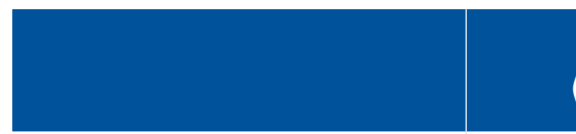

Age at diagnosis, median (IOR)

$68(60-76)$

\section{No LN \\ ( $n=15468)$}

LNI

( $n=20767$ )

$69(61-76)$

( $n=5299$ )

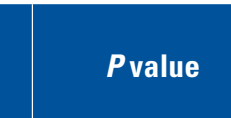

Sex, n (\%)

$$
\text { Male }
$$

Female

15705 (75.6)

5062 (24.4)

11844 (76.6)

3624 (23.4)

3861 (72.9)

$<0.001$

$68(60-75)$

$<0.001$

\section{Race, $\mathrm{n}(\%)$ \\ White \\ Black \\ Hispanic \\ Asiatic \\ Other}

$17089(82.3)$
$1260(6.1)$
$1359(6.5)$
$944(4.5)$
$115(0.6)$

12811 (82.8)

$873(5.6)$

984 (6.4)

712 (4.6)

1438 (27.1)

$88(0.6)$

$4278(80.7)$

387 (7.3)

375 (7.1)

232 (4.4)

27 (0.5)

\section{Socioeconomical status, $\mathrm{n}(\%)$}

High

Low

10385 (50.1)

10382 (49.9)

$7730(50)$

$7738(50)$

$2655(50.1)$

2644 (49.9)

\begin{tabular}{|c|c|}
\hline $8363(54.1)$ & $2854(53.9)$ \\
\hline $1804(11.7)$ & $572(10.8)$ \\
\hline $2674(17.3)$ & $904(17.1)$ \\
\hline $2627(17.0)$ & $969(18.3)$ \\
\hline
\end{tabular}

Region, n (\%)

West

Midwest

North-East

South

$11217(54.0)$
$2376(11.4)$
$3578(17.2)$
$3596(17.3)$

18303 (88.1)

$177(0.9)$

$38(0.2)$

$283(1.4)$

592 (2.9)

$85(0.4)$

889 (4.3)

382 (1.8)

$18(0.1)$
13707 (88.6)

$89(0.6)$

$27(0.2)$

211 (1.4)

417 (2.7)

$68(0.4)$

669 (4.3)

$265(1.7)$

$15(0.1)$
4596 (86.7)

$88(1.7)$

$11(0.2)$

$72(1.4)$

175 (3.3)

$17(0.3)$

220 (4.2)

$117(2.2)$

$3(0.1)$

\section{Marital status, n (\%)}

Married

$13242(63.8)$
$2339(11.3)$
$4440(21.4)$
$746(3.6)$

$9983(64.5)$
$1683(10.9)$
$3226(20.9)$
$576(3.7)$

3259 (61.5)

$656(12.4)$

1214 (22.9)

Previously married

Unknown

746 (3.6)

576 (3.7)

$170(3.2)$

CT: chemotherapy; IQR: interquartile range; LN: Iymph node; LNI: lymph node invasion; NNR: number of lymph nodes removed; TMT: trimodal therapy; TURB: transurethral resection of the bladder; UC: urothelial carcinoma 
TABLE 2.

Descriptive characteristics according to the lymph node invasion status in the study population $(\mathrm{n}=20767)$, Cont'd
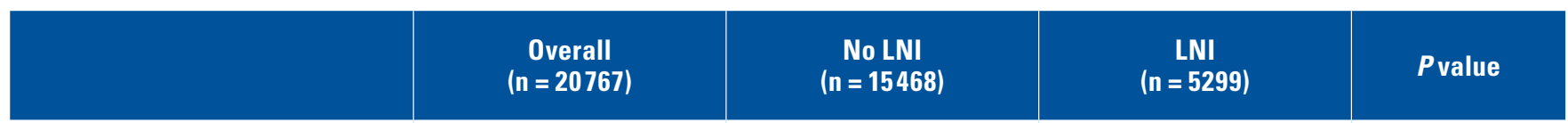

Treatment, n (\%)

Radical cystectomy

Partial cystectomy

TURB only

Radiotherapy

TMT

No local treatment

Other/Unknown

$18125(87.3)$
$1044(5)$
$739(3.6)$
$9(0)$
$119(0.6)$
$49(0.2)$
$682(3.3)$

$13677(88.4)$
$911(5.9)$
$473(3.1)$
$2(0)$
$40(0.3)$
$19(0.1)$
$346(2.2)$

4448 (83.9)

$133(2.5)$

$266(5.0)$

$7(0.1)$

$79(1.5)$

$30(0.6)$

336 (6.3)

\section{Perioperative CT, n (\%)}

Yes

No/Unknown

\section{6 (38.6)}

12761 (61.4)

5087 (32.9)

10381 (67.1)

$2919(55.1)$

$<0.001$

T stage, $\mathrm{n}(\%)$

TO/x

$\mathrm{T}<2$

T2

T3-4

$138(0.7)$
$2786(13.4)$
$7333(35.3)$
$10510(50.6)$

87 (0.6)

2628 (17.0)

$6325(40.9)$

2380 (44.9)

$<0.001$

$$
6428 \text { (41.6) }
$$

$51(1.0)$
$158(3.0)$
$1008(19.0)$
$4082(77.0)$

\section{Grade, n (\%)}

G1

G2

G3

G4

Gx

195 (0.9)

$1116(5.4)$

6385 (30.7)

12007 (57.8)

1064 (5.1)

167 (1.1)

$936(6.1)$

4579 (29.6)

8949 (57.9)

$28(0.5)$

180 (3.4)

1806 (34.1)

3058 (57.7)

837 (5.4)

227 (4.3)

NNR, median (IOR)

$12(6-22)$

$12(6-22)$

$13(6.5-21)$

0.4

Positive LN, median (IQR)

$0(0-1)$

$0(0)$

$2(1-4)$

$<0.001$

CT: chemotherapy; IQR: interquartile range; LN: Iymph node; LNI: lymph node invasion; NNR: number of lymph nodes removed; TMT: trimodal therapy; TURB: transurethral resection of the bladder; UC: urothelial carcinoma 


\section{TABLE 3.}

Univariable and multivariable logistic regression analysis predicting lymph node invasion in the overall population $(n=20767)$
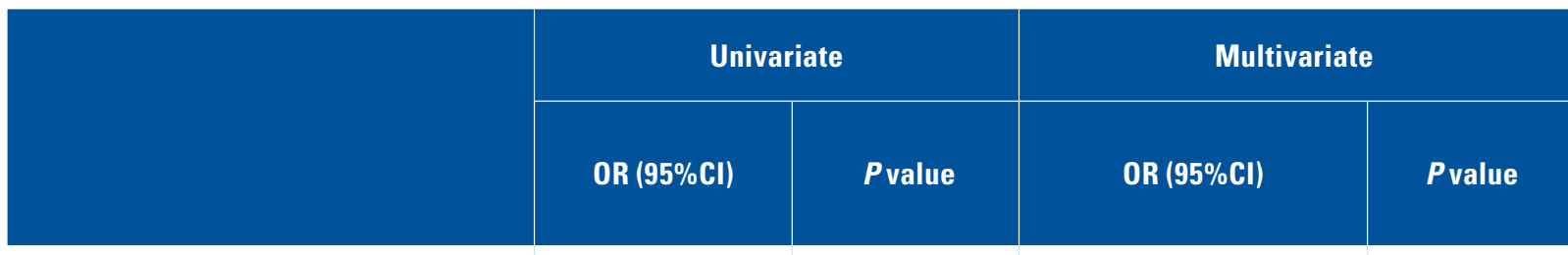

\section{Histology}

Urothelial carcinoma

Micropapillary UC

Lymphoepithelioma-like UC

\begin{tabular}{c|c}
\hline $2.95(2.19-3.97)$ & $<0.001$ \\
\hline $1.22(0.58-2.39)$ & 0.6 \\
\hline $1.02(0.77-1.33)$ & 0.9 \\
$1.25(1.05-1.50)$ & 0.01 \\
\hline $0.75(0.42-1.24)$ & 0.3 \\
$1.32(1.06-1.64)$ & 0.01 \\
\hline $0.98(0.84-1.14)$ & 0.8 \\
\hline $0.60(0.14-1.81)$ & 0.4
\end{tabular}

Reference

Sarcomatoid UC

Adenocarcinoma

Giant and spindle cell carcinoma

Neuroendocrine tumor

Squamous cell carcinoma

Sarcoma

$0.60(0.14-1.81)$

0.4

$3.39(2.43-4.74)$
$0.84(0.38-1.76)$
$0.89(0.66-1.18)$
$1.22(0.99-1.50)$
$0.60(0.33-1.03)$
$1.04(0.81-1.32)$
$0.90(0.75-1.08)$
$0.45(0.10-1.48)$

$<0.00$

0.7

0.4

0.06

0.07

0.8

0.3

0.2

Age at diagnosis (years)

$0.99(0.99-1.00)$

$<0.001$

$0.99(0.99-1.00)$

0.04

1

\section{Sex}

Male

Female

$1.22(1.14-1.31)$

$<0.001$

Reference

1.12 (1.03-1.22)

0.006

0.2

\section{Race}

White

Black

Hispanic

Asiatic

Other

\begin{tabular}{c|c}
- & $<0.001$ \\
\hline $1.32(1.17-1.50)$ & 0.04 \\
$1.14(1.01-1.29)$ & 0.7 \\
$0.98(0.84-1.13)$ & 0.7
\end{tabular}

Reference

1.17 (1.01-1.34)

$1.16(1.01-1.34)$

$0.98(0.83-1.16)$

$0.85(0.52-1.35)$

Reference

Low

High

$1.01(0.94-1.07)$

0.9

0.99 (0.92-1.06)

0.7

\section{Region}

West

Midwest

North-East

South

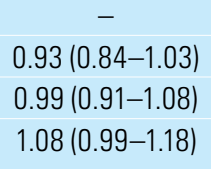

0.2
0.8
0.07

Reference

$0.84(0.74-0.94)$

$0.93(0.84-1.02)$

$1.02(0.92-1.12)$

0.1

0.1

1

0.7

0.03
0.03
0.8
0.5

1

1

1

1

\section{Marital status}

Married

Never married

Previously married

Unknown

\begin{tabular}{c|c|}
\hline $1.19(1.08-1.32)$ & $<0.001$ \\
\hline $1.15(1.07-1.25)$ & $<0.001$ \\
\hline $0.90(0.76-1.08)$ & 0.3 \\
\hline
\end{tabular}

Reference

$1.13(1.01-1.26)$

$1.12(1.03-1.23)$

$1.04(0.86-1.26)$

0.03
0.008
0.7

1

0.3

0.3

Cl: confidence interval; CT: chemotherapy; OR: odd ratio; NNR: number of lymph nodes removed; UC: urothelial carcinoma

${ }^{*} P$ values were adjusted using the conservative Bonferroni correction method which multiplies the raw $P$-values by the number of tests 


\section{TABLE 3.}

Univariable and multivariable logistic regression analysis predicting lymph node invasion in the overall population $(\mathrm{n}=20767)$, Cont'd

\begin{tabular}{|c|c|c|c|c|c|}
\hline & \multicolumn{2}{|c|}{ Univariate } & \multicolumn{2}{|c|}{ Multivariate } & \multirow[b]{2}{*}{$\begin{array}{l}\text { Adjusted } \\
\text { Pvalue* }\end{array}$} \\
\hline & OR (95\%CI) & Pvalue & OR (95\%CI) & Pvalue & \\
\hline \multicolumn{6}{|l|}{ Perioperative CT } \\
\hline No & - & & Reference & & \\
\hline Yes & $2.50(2.35-2.67)$ & $<0.001$ & $2.29(2.13-2.46)$ & $<0.001$ & $<0.001$ \\
\hline \multicolumn{6}{|l|}{ Treatment } \\
\hline No local treatment & - & & Reference & & \\
\hline Radical cystectomy & $0.21(0.11-0.36)$ & $<0.001$ & 0.18 (0.09-0.34) & $<0.001$ & $<0.001$ \\
\hline Partial cystectomy & $0.09(0.05-0.17)$ & $<0.001$ & $0.09(0.04-0.18)$ & $<0.001$ & $<0.001$ \\
\hline TURB only & $0.36(0.19-0.63)$ & $<0.001$ & 0.57 (0.29-1.07) & 0.09 & 1 \\
\hline Radiotherapy & $2.21(0.48-15.9)$ & 0.4 & $1.42(0.27-11.23)$ & 0.7 & 1 \\
\hline TMT & $1.25(0.62-2.48)$ & 0.5 & $0.76(0.35-1.61)$ & 0.5 & 1 \\
\hline Other/Unknown & $0.62(0.33-1.11)$ & 0.1 & $0.32(0.16-0.62)$ & $<0.001$ & 0.03 \\
\hline \multicolumn{6}{|l|}{ T stage } \\
\hline$T<2$ & - & & Reference & & \\
\hline $\mathrm{T} 2$ & $2.65(2.23-3.16)$ & $<0.001$ & 2.24 (1.88-2.69) & $<0.001$ & $<0.001$ \\
\hline T3-4 & $10.6(8.98-12.5)$ & $<0.001$ & $9.44(7.98-11.2)$ & $<0.001$ & $<0.001$ \\
\hline $\mathrm{TO} / \mathrm{x}$ & $9.74(6.63-14.2)$ & $<0.001$ & $6.21(4.08-9.35)$ & $<0.001$ & $<0.001$ \\
\hline \multicolumn{6}{|l|}{ Grade } \\
\hline G1 & - & & Reference & & \\
\hline $\mathrm{G} 2$ & $1.15(0.76-1.80)$ & 0.5 & $0.78(0.50-1.27)$ & 0.3 & 1 \\
\hline G3 & $2.35(1.60-3.60)$ & $<0.001$ & $1.50(0.98-2.37)$ & 0.07 & 1 \\
\hline G4 & 2.04 (1.39-3.11) & $<0.001$ & $1.33(0.87-2.10)$ & 0.2 & 1 \\
\hline$G x$ & $1.62(1.07-2.52)$ & 0.03 & $1.16(0.74-1.87)$ & 0.5 & 1 \\
\hline NNR & $1.00(0.99-1.00)$ & 0.5 & 1.00 (0.99-1.00) & 0.3 & 1 \\
\hline
\end{tabular}

Cl: confidence interval; CT: chemotherapy; OR: odd ratio; NNR: number of lymph nodes removed; UC: urothelial carcinoma

${ }^{*} P$ values were adjusted using the conservative Bonferroni correction method which multiplies the raw $P$-values by the number of tests 


\section{TABLE 4.}

Univariable and multivariable Cox regression analyses predicting overall mortality among patients with lymph node invasion $(n=5299)$

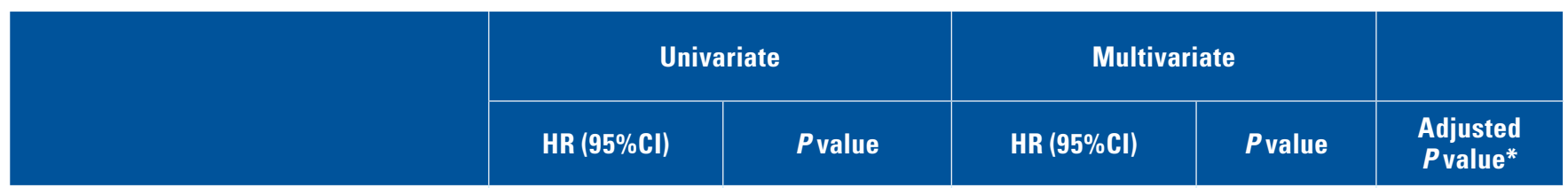

\section{Histology}

Urothelial carcinoma

Micropapillary UC

Lymphoepithelioma-like UC

Sarcomatoid UC

Adenocarcinoma

Giant and spindle cell carcinoma

Neuroendocrine tumor

Squamous cell carcinoma

Sarcoma

\begin{tabular}{|c|c|}
\hline $0.97(0.72-1.30)$ & 0.84 \\
\hline $0.46(0.11-1.82)$ & 0.27 \\
\hline $1.55(1.16-2.08)$ & $<0.001$ \\
\hline $1.17(0.97-1.42)$ & 0.10 \\
\hline $0.69(0.29-1.66)$ & 0.40 \\
\hline $1.33(1.06-1.68)$ & 0.01 \\
\hline $2.03(1.72-2.40)$ & $<0.001$ \\
\hline $4.69(1.17-18.8)$ & 0.03 \\
\hline
\end{tabular}

Reference

\begin{tabular}{|c|c|c|}
\hline $1.09(0.81-1.47)$ & 0.55 & 1 \\
\hline $0.47(0.12-1.87)$ & 0.28 & 1 \\
\hline $1.37(1.02-1.84)$ & 0.04 & 1 \\
\hline $1.10(0.90-1.34)$ & 0.36 & 1 \\
\hline $0.67(0.28-1.60)$ & 0.36 & 1 \\
\hline $1.34(1.06-1.70)$ & 0.01 & 0.35 \\
\hline $1.87(1.56-2.24)$ & $<0.001$ & $<0.001$ \\
\hline $4.84(1.21-19.4)$ & 0.03 & 1 \\
\hline
\end{tabular}

$1.01(1.01-1.01)$

$<0.001$

$<0.001$

\section{Age at diagnosis (years)}

1.01 (1.01-1.01)

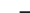

\section{Male}

Female

\section{Race}

White

Black

Hispanic

Asiatic

Other

\section{$1.12(1.04-1.21)$}

$$
<0.001
$$

Reference

$1.04(0.96-1.13)$

0.37

1

Reference

\begin{tabular}{|c|c|}
\hline $1.27(1.12-1.45)$ & $<0.001$ \\
$0.93(0.81-1.08)$ & 0.33 \\
\hline $0.97(0.82-1.15)$ & 0.76 \\
\hline $1.02(0.62-1.67)$ & 0.94
\end{tabular}

$1.18(1.03-1.36)$

$0.97(0.84-1.13)$

$1.04(0.88-1.24)$

$1.04(0.63-1.70)$

0.73

0.65

0.89

\section{Reference}

$1.00(0.93-1.08)$

0.96

1

Low

High

1.01 (0.94-1.08)

0.77

\section{Reference}

West

Midwest

North-East

$1.07(0.95-1.21)$

0.23

$1.15(1.04-1.27)$

$<0.001$

$1.28(1.16-1.41)$

$<0.001$

$1.10(0.97-1.25)$
$1.05(0.95-1.17)$
$1.22(1.10-1.35)$

$1.22(1.10-1.35)$

0.12
0.33
$<0.001$

1

1

$<0.001$

\section{Marital status}

Married

Never married

Previously married

Unknown

\begin{tabular}{c|c}
$1.27(1.14-1.41)$ & $<0.001$ \\
\hline $1.21(1.11-1.32)$ & $<0.001$ \\
\hline $0.94(0.76-1.18)$ & 0.61
\end{tabular}

Reference

\begin{tabular}{c|c|c}
\hline $1.24(1.11-1.39)$ & $<0.001$ & $<0.001$ \\
\hline $1.10(1.01-1.21)$ & 0.03 & 1 \\
\hline $0.90(0.71-1.12)$ & 0.34 & 1 \\
\hline
\end{tabular}

Cl: confidence interval; CT: chemotherapy; HR: hazard ratio; NNR: number of lymph nodes removed; TMT: trimodal therapy;

TURB: transurethral resection of the bladder: UC: urothelial carcinoma

* $P$ values were adjusted using the conservative Bonferroni correction method which multiplies the raw $P$-values by the number of tests 
TABLE 4.

Univariable and multivariable Cox regression analyses predicting overall mortality among patients with lymph node invasion $(n=5299)$, Cont'd
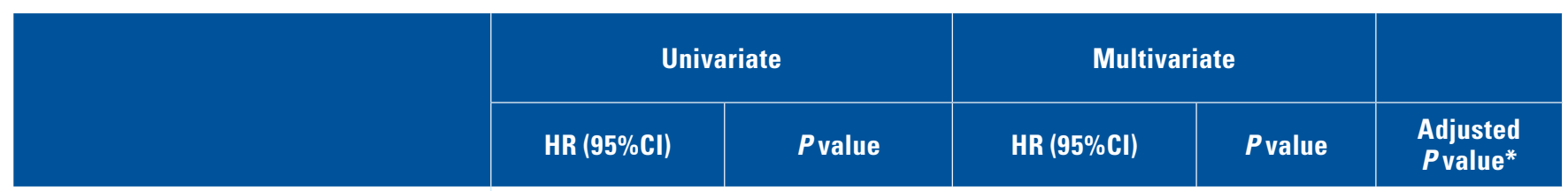

\section{Perioperative CT}

No

Yes

Reference

$0.69(0.64-0.74)<0.001<0.001$

\section{Treatment}

No local treatment

Radical cystectomy

Partial cystectomy

TURB only

Radiotherapy

TMT

Other/Unknown

$\begin{array}{cc}- & \\ 0.77(0.46-1.27) & 0.30 \\ 0.67(0.38-1.16) & 0.15 \\ 0.92(0.54-1.56) & 0.76 \\ 3.08(1.19-7.93) & 0.02 \\ 0.82(0.46-1.46) & 0.51 \\ 0.94(0.56-1.59) & 0.82\end{array}$

\section{Reference}

$0.83(0.48-1.45)$

$0.65(0.36-1.18)$

$1.12(0.63-1.98)$

$3.82(1.46-9.98)$

$1.13(0.61-2.09)$

$0.98(0.55-1.75)$

\section{T stage}

$\mathrm{T}<2$

T2

T3-4

$\mathrm{TO} / \mathrm{x}$

$\begin{array}{cc}1.05(0.82-1.35) & 0.70 \\ 1.83(1.45-2.33) & <0.001 \\ 1.64(1.04-2.56) & 0.03\end{array}$

Reference

$\begin{array}{ccc}1.12(0.87-1.45) & 0.37 & 1 \\ 1.94(1.52-2.47) & <0.001 & 0.03 \\ 1.32(0.81-2.15) & 0.27 & 1\end{array}$

Grade
G1
G2
G3
G4
GX

NNR

$0.99(0.99-0.99)$

\section{Reference}

$\begin{array}{lll}0.25 & 0.95(0.56-1.62) & 0.86 \\ 0.59 & 1.05(0.64-1.72) & 0.85 \\ 0.99 & 0.99(0.61-1.63) & 0.98 \\ 0.78 & 0.98(0.58-1.66) & 0.94\end{array}$

$0.99(0.99-0.99) \quad<0.001$

$<0.001$

Cl: confidence interval; CT: chemotherapy; HR: hazard ratio; NNR: number of lymph nodes removed; TMT: trimodal therapy;

TURB: transurethral resection of the bladder; UC: urothelial carcinoma

* $P$ values were adjusted using the conservative Bonferroni correction method which multiplies the raw $P$-values by the number of tests 


\section{References}

1. Ku JH, Kang M, Kim HS, Jeong CW, Kwak C, Kim HH. Lymph node density as a prognostic variable in node-positive bladder cancer: a meta-analysis. BMC Cancer.2015;15:447.

2. Bandini M, Briganti A, Plimack ER, Niegisch G, Yu EY, Bamias A, et al. Modeling 1-year relapse-free survival after neoadjuvant chemotherapy and radical cystectomy in patients with clinical T2-4N0MO urothelial bladder carcinoma: endpoints for phase 2 trials. Eur Urol Oncol.2019;2(3):248-256. doi: 10.1016/j.euo.2018.08.009

3. Pederzoli F, Bandini M, Briganti A, Plimack ER, Niegisch G, Yu EY, et al. Incremental utility of adjuvant chemotherapy in muscleinvasive bladder cancer: quantifying the relapse risk associated with therapeutic effect. Eur Urol.2019;76(4):425-429. doi: 10.1016/j. eururo.2019.06.032

4. Zaffuto E, Bandini M, Moschini M, Leyh-Bannurah S-R, Gazdovich $S$, Dell'Oglio P, et al. Location of metastatic bladder cancer as a determinant of in-hospital mortality after radical cystectomy. Eur Urol Oncol.2018;1(2):169-175. doi: 10.1016/j.euo.2018.02.001

5. Witjes JA, Bruins HM, Cathomas R, Compérat EM, Cowan NC, Gakiset $\mathrm{G}$, al. European Association of Urology guidelines on muscle-invasive and metastatic bladder cancer: summary of the 2020 guidelines. Eur Urol.2021;79(1):82-104. doi: 10.1016/j.eururo.2020.03.055

6. Zaffuto E, Bandini M, Gazdovich S, Valiquette AS, Leyh-Bannurah SR, Tian Z, al. Contemporary rates of adherence to international guidelines for pelvic lymph node dissection in radical cystectomy: a population-based study. World J Urol.2018;36(9):1417-1422. 10.1007/ s00345-018-2306-7

7. Witjes JA, Babjuk M, Bellmunt J, Bruins HM, De Reijke TM, De Santiset $\mathrm{M}$,al. EAU-ESMO consensus statements on the management of advanced and variant bladder cancer - an international collaborative multistakeholder effortt. Eur Urol.2020;77(2):223-250. doi: 10.1016/j. eururo.2019.09.035

8. Burger M, Kamat AM, McConkey D. Does variant histology change management of non-muscle-invasive bladder cancer? Eur Urol Oncol.2021 Jun;4(3):510-514. doi: 10.1016/j.euo.2019.06.0129.

9. Alanee S, Alvarado-Cabrero I, Murugan P, Kumar R, Nepple KG, Paner $\mathrm{GP}$, et al. Update of the International Consultation on Urological Diseases on bladder cancer 2018: non-urothelial cancers of the urinary bladder. World J Urol.2019;37(1):107-114. doi: 10.1007/ s00345-018-2421-5

10. Pederzoli F, Bandini M, Marandino L, Ali SM, Madison R, Chung J, et al. Targetable gene fusions and aberrations in genitourinary oncology. Nat Rev Urol.2020;17(11):613-625. doi: 10.1038/s41585-020-00379-4

11. Basile G, Pederzoli F, Bandini M, Raggi D, Gallina A, Salonia A, et al. Intermediate- and high-risk nonmuscle invasive bladder cancer: where do we stand? Urol Oncol.2021 Mar 22;S1078-1439(21)00081-8. doi: 10.1016/j.urolonc.2021.02.020.

12. Humphrey PA, Moch H, Cubilla AL, Ulbright TM, Reuter VE. The 2016 WHO Classification of Tumours of the Urinary System and Male Genital Organs-Part B: Prostate and Bladder Tumours. Eur Urol.2016;70(1):106-119.
13. Hoffman-Censits J, Choi W, Pal S, Trabulsi E, Kelly WK, Hahn NM, et al. Urothelial cancers with small cell variant histology have confirmed high tumor mutational burden, frequent TP53 and RB Mutations, and a unique gene expression profile. Eur Urol Oncol.2021 Apr;4(2):297-300. Epub 2020 Feb 12. doi:10.1016/j.euo.2019.12.002

14. Stensland KD, Zaid H, Broadwin M, Sorcini A, Canes D, Galsky M, et al. Comparative effectiveness of treatment strategies for squamous cell carcinoma of the bladder. Eur Urol Oncol.2020 Aug;3(4):509-514. Epub 2018 Dec 1. doi: 10.1016/j.euo.2018.11.003

15. NIH National Cancer Institute. Citations for SEER Databases and SEER ${ }^{*}$ Stat Software. SEER n.d. Available at: https://seer.cancer.gov/ data/citation.html. Accessed July 31, 2021.

16. Luzzago S, Palumbo C, Rosiello G, Knipper S, Pecoraro A, Deuker M, et al. Survival after partial cystectomy for variant histology bladder cancer compared with urothelial carcinoma: a population-based study. Clin Genitourin Cancer.2020;18(2):117-128.e5. https://doi. org/10.1016/j.clgc.2019.10.016

17. Bandini M, Marchioni M, Pompe RS, Tian Z, Gandaglia G, Fossati N, et al. First North American validation and head-to-head comparison of four preoperative nomograms for prediction of lymph node invasion before radical prostatectomy. BJU Int.2018;121(4):592-599. Epub 2017 Nov 29. doi: 10.1111/bju.14074

18. Seisen $T$, Compérat $E$, Léon $P$, Roupret M. Impact of histological variants on the outcomes of nonmuscle invasive bladder cancer after transurethral resection. Curr Opin Urol.2014;24(5):524-531.

19. Babjuk M, Burger M, Compérat E, Gontero P, Liedberg F, MassonLecomte A, et al. EAU guidelines on non-muscle-invasive bladder cancer (TaT1 and CIS) 2018. In: European Association of Urology Guidelines. 2018 Edition. Vol presented at the EAU Annual Congress Copenhagen 2018. European Association of Urology Guidelines Office; 2018.Available at: https://uroweb.org/guideline/non-muscle-invasivebladder-cancer/\#11. Accessed July 31, 2021.

20. Veskimäe E, Espinos EL, Bruins HM, Yuan Y, Sylvester R, Kamat AM, et al. What is the prognostic and clinical importance of urothelial and nonurothelial histological variants of bladder cancer in predicting oncological outcomes in patients with muscle-invasive and metastatic bladder cancer? A European Association of Urology muscle invasive and metastatic bladder cancer guidelines panel systematic review. Eur Urol Oncol.2019;2(6):625-642. Epub 2019 0ct 8. doi: 10.1016/j. euo.2019.09.003

21. Deuker M, Stolzenbach LF, Collà Ruvolo C, Nocera L, Mansour M, Tian Z, et al. Micropapillary versus urothelial carcinoma of the urinary bladder: stage at presentation and efficacy of chemotherapy across all stages-A SEER-based study. Eur Urol Focus.2020 Sep 19;S24054569(20)30223-6. doi: 10.1016/j.euf.2020.08.010

22. Bruins HM, Veskimae E, Hernandez V, Imamura M, Neuberger MM, Dahm $\mathrm{P}$, et al. The impact of the extent of lymphadenectomy on oncologic outcomes in patients undergoing radical cystectomy for bladder cancer: a systematic review. Eur Urol.2014;66(6):1065-1077. Epub 2014 Jul 26.doi: 10.1016/j.eururo.2014.05.031 
23. Larcher A, Sun M, Schiffmann J, Tian Z, Shariat SF, McCormack M, et al. Differential effect on survival of pelvic lymph node dissection at radical cystectomy for muscle invasive bladder cancer. Eur $J$ Surg Oncol.2015;41(3):353-360. Epub 2014 Nov 21. doi: 10.1016/j. ejso.2014.10.061

24. Perera M, McGrath S, Sengupta S, Crozier J, Bolton D, Lawrentschuk N. Pelvic lymph node dissection during radical cystectomy for muscleinvasive bladder cancer. Nat Rev Urol.2018;15(11):686-692. doi: 10.1038/s41585-018-0066-1

25. Kim SP, Frank I, Cheville JC, Thompson RH, Weight CJ, et al. The Impact of squamous and glandular differentiation on survival after radical cystectomy for urothelial carcinoma. J Urol.2012;188(2):405-409. Epub 2012 Jun 14. doi: 10.1016/j.juro.2012.04.020

26. Linder BJ, Frank I, Cheville JC, Thompson RH, Thapa P, Tarrell RF, et al. Outcomes following radical cystectomy for nested variant of urothelial carcinoma: a matched cohort analysis. J Urol.2013 May;189(5):16701675. Epub 2012 Nov 6. doi: 10.1016/j.juro.2012.11.006

27. Naspro R, Finati M, Roscigno M, Pellucchi F, La Croce G, Sodano M, et al. The impact of histological variants on outcomes after open radical cystectomy for muscle-invasive urothelial bladder cancer: results from a single tertiary referral centre. World J Urol.2021 Jun;39(6):19171926. Epub 2020 Jul 21. doi: 10.1007/s00345-020-03364-z

28. Marks P, Gild P, Soave A, Janisch F, Minner S, Engel O, et al. The impact of variant histological differentiation on extranodal extension and survival in node positive bladder cancer treated with radical cystectomy. Surg Oncol.2019;28:208-213. Epub 2019 Jan 30. doi: 10.1016/j.suronc.2019.01.008

29. Nassar H, Pansare V, Zhang H, Che M, Sakr W, Ali-Fehmi R, et al. Pathogenesis of invasive micropapillary carcinoma: role of MUC1 glycoprotein. Mod Pathol.2004;17(9):1045-1050. doi: 10.1038/ modpathol.3800166

30. Nassar H. Carcinomas with micropapillary morphology: clinical significance and current concepts. Adv Anat Pathol.2004;11(6):297-303.

31. Pederzoli F, Bandini M, Marandino L, Raggi D, Giannatempo P, Salonia A, et al. Neoadjuvant chemotherapy or immunotherapy for clinical T2N0 muscle-invasive bladder cancer: time to change the paradigm? Eur Urol Oncol.2020 Aug 23;S2588-9311(20)30121-8. doi: 10.1016/j. euo.2020.07.006.32
32. Bandini M, Gibb EA, Gallina A, Raggi D, Marandino L, Bianchi M, et al. Does the administration of preoperative pembrolizumab lead to sustained remission post-cystectomy? First survival outcomes from the PURE-01 study گ. Ann Oncol.2020;31(12):1755-1763. Epub 2020 Sep 23. doi: 10.1016/j.annonc.2020.09.011

33. Bandini M, Pederzoli F, Madison R, Briganti A, Ross JS, Niegisch $G$, et al. Unfavorable cancer-specific survival after neoadjuvant chemotherapy and radical cystectomy in patients with bladder cancer and squamous cell variant: a multi-institutional study. Clin Genitourin Cancer.2020;18(5):e543-e556. Epub 2020 Feb 8. doi: 10.1016/j. clgc.2020.01.007

34. Basile G, Bandini M, Pederzoli F, Gallina A, Necchi A. Re: Siamak Daneshmand, Azadeh Nazemi. Neoadjuvant Chemotherapy in variant histology bladder cancer: current evidence. Eur Urol Focus.2020;6:639-41.

35. Necchi A, Pederzoli F, Bandini M, Spiess PE. Revolutionizing care for rare genitourinary tumours. Nat Rev Urol.2021 Feb;18(2):69-70. doi: $10.1038 / s 41585-020-00402-8$

36. Bandini M, Preisser F, Nazzani S, Marchioni M, Tian Z, Fossati N, et al. Contemporary trends and survival outcomes after aborted radical prostatectomy in lymph node metastatic prostate cancer patients. Eur Urol Focus.2019;5(3):381-388. Epub 2018 Feb 1. doi: 10.1016/j. euf.2018.01.009

37. Moch, H M, Humphrey P, Ulbright T, Reuter V. WHO Classification of Tumours of the Urinary System and Male Genital Organs. International Agency for Research on Cancer; 2016.

38. Linder BJ, Boorjian SA, Cheville JC, Sukov WR, Thapa P, Tarrell RF, et al. The impact of histological reclassification during pathology re-review-evidence of a Will Rogers effect in bladder cancer? J Urol.2013;190(5):1692-1696. Epub 2013 May 23. doi: 10.1016/j. juro.2013.05.040

39. Shah RB, Montgomery JS, Montie JE, Kunju LP. Variant (divergent) histologic differentiation in urothelial carcinoma is under-recognized in community practice: Impact of mandatory central pathology review at a large referral hospital. Urol Oncol.2013;31(8):1650-1655. Epub 2012 May 17. doi: 10.1016/j.urolonc.2012.04.009 